\title{
On the control of Markov jump linear systems with no mode observation: application to a DC Motor device
}

\author{
Alessandro N. Vargas ${ }^{1, *}$, Eduardo F. Costa ${ }^{2}$ and João B. R. do Val ${ }^{3}$ \\ ${ }^{1}$ Universidade Tecnológica Federal do Paraná, UTFPR, \\ Av. Alberto Carazzai 1640, 86300-000 Cornelio Procópio-PR, Brazil. \\ ${ }^{1}$ Basque Center for Applied Mathematics, BCAM, \\ Alameda de Mazarredo 14, E-48009 Bilbao, Vizcaya, Spain. \\ ${ }^{2}$ Universidade de São Paulo, USP, \\ Depto. Matemática Aplicada e Estatística, C.P. 668, 13560-970, São Carlos-SP, Brazil. \\ ${ }^{3}$ Universidade Estadual de Campinas, UNICAMP, \\ FEEC-DT, Av. Albert Einstein 400, 13083-852 Campinas-SP, Brazil.
}

\begin{abstract}
SUMMARY
This paper deals with the control problem of discrete-time Markov jump linear systems for the case in which the controller does not have access to the state of the Markov chain. A necessary optimal condition, which is nonlinear with respect to the optimizing variables, is introduced and the corresponding solution is obtained through a variational convergent method. We illustrate the practical usefulness of the derived approach by applying it in the control speed of a real DC Motor device subject to abrupt power failures. Copyright (C) 2011 John Wiley \& Sons, Ltd.
\end{abstract}

Received ...

KEY WORDS: Markov jump linear systems; stochastic systems; control of DC Motor.

\section{INTRODUCTION}

The class of systems known as Markov Jump Linear Systems (MJLS) has been intensively investigated over the last two decades, mainly due to the fact that they are suitable for modelling, control and estimation of physical processes that are subject to abrupt changes. For instance, the MJLS approach is applied in the control of paper mills [15], robotics [24, 27], economy [6, 9], networks [14], to cite a few. Regarding recent theoretical developments, we can cite the papers $[3,4,5,10,11,13,25]$, and the monograph [7], as a small sample. Notwithstanding the large number of contributions on control of MJLS, most of the available results deal with the case in which the controller has complete access to the Markov state. Even in the context of partial information, most results focus on the jump mode observation, see for instance [8] and [12]. In practice, this signifies that the controller has a built-in sensor or a similar measurement instrument that determines exactly and instantaneously, at each instant of time, the active jump mode. However, such a device can be costly or it may not be even feasible. In principle, optimal control in the situation with no mode

${ }^{*}$ Correspondence to: Alessandro N. Vargas, UTFPR, Av. Alberto Carazzai 1640, 86300-000 Cornelio Procópio-PR, Brazil. E-mail: avargas@utfpr.edu.br

Contract/grant sponsor: Brazilian agencies FAPESP and CNPq; and Spanish agency Fundación Carolina; contract/grant number: FAPESP 03/06736-7, 04/06947-0; CNPq 471557/2009-9, 304856/2007-0, 304429/2007-4, 306466/2010-4; and Fundación Carolina - Programa "Movilidad de profesores e investigadores Brasil-España. C.2010".

Copyright @ 2011 John Wiley \& Sons, Ltd.

Prepared using rncauth.cls [Version: 2010/03/27 v2.00] 
observation can be dealt with the theory of dynamic programming with imperfect state information, however, for the problem we are dealing with, this would lead to a nonlinear and high-dimensional optimization problem involving the information vector, also called the information state [2], [17]. Thus, it is reasonable to consider a control policy that is not a function of the active jump mode, and minimizes a suitable quadratic performance index. This is the scenario under investigation in this paper. The results can be easily extended to the scenario of cluster observation of the jump mode, as explained in Remark 3.5. The derived results are verified in an application of a DC Motor device, see Section 4 in connection.

The setup of MJLS with no mode observation is studied in the papers [9], [10], and [20], and in the monograph [7, Ch. 3.5.2]. Notice that all of these approaches do not consider additive noise input, i.e., it is assumed that $w_{k} \equiv 0$. The paper [9] presents a necessary optimal condition for the control problem in the receding horizon context with no noise; the papers [10] and [20] deal with the $\mathrm{H}_{2}$ control problem but the techniques based on LMI assure a guaranteed cost only; the monograph [7, Ch. 3.5.2] considers the stabilisation problem taking the MJLS with no noise. Our results expand the knowledge of MJLS with additive noise input, and in this noisy setup, a method to compute the necessary optimal condition for the corresponding control problem is obtained. The main result is illustrated by a real application to a DC Motor apparatus.

The MJLS considered in this paper is as follows. Let $\left(\Omega, \mathcal{F},\left\{\mathcal{F}_{k}\right\}, P\right)$ be a fixed filtered probability space, and consider the system

$$
x_{k+1}=A_{\theta_{k}} x_{k}+B_{\theta_{k}} u_{k}+H_{\theta_{k}} w_{k}, \quad \forall k \geq 0, x_{0} \in \mathcal{R}^{r}, \theta_{0} \sim \pi_{0},
$$

where $x_{k}, u_{k}$, and $w_{k}, k \geq 0$ are processes taking values respectively in $\mathcal{R}^{r}, \mathcal{R}^{s}$, and $\mathcal{R}^{q}$. The noisy input $\left\{w_{k}\right\}$ forms an iid process with zero mean and covariance matrix equals to the identity for all $k \geq 0$, and the process $\left\{\theta_{k}\right\}$ represents a discrete-time homogeneous Markov chain. The state of the system is formed by the pair $\left(x_{k}, \theta_{k}\right)$, and $u_{k}$ is the control. The matrices $A_{\theta_{k}}, B_{\theta_{k}}$, and $H_{\theta_{k}}, k \geq 0$, have compatible dimensions. To measure the performance of the system (1), we consider a standard $N$-th horizon quadratic cost

$$
J_{N}\left(x_{0}, \pi_{0}\right):=\mathrm{E}_{x_{0}, \pi_{0}}\left[\sum_{k=0}^{N-1}\left(x_{k}^{\prime} Q_{\theta_{k}} x_{k}+u_{k}^{\prime} R_{\theta_{k}} u_{k}\right)+x_{N}^{\prime} F_{\theta_{N}} x_{N}\right],
$$

where $\mathrm{E}_{x_{0}, \pi_{0}}[\cdot] \equiv \mathrm{E}\left[\cdot \mid x_{0}, \pi_{0}\right]$ represents the expected value operator, and $Q_{\theta_{k}}, R_{\theta_{k}}$, and $F_{\theta_{k}}, k \geq 0$, are given matrices. We assume that only the variable $x_{k}$ is observed at time instant $k$, and we consider that the controller is in the linear state-feedback form [7, p. 59], [9],

$$
u_{k}=G(k) x_{k}, \quad k \geq 0 .
$$

Note in (3) that the gain matrix $G(k)$ does not depend on the Markov state $\theta_{k}$ nor on the conditional distribution $P\left(\theta_{k} \mid x_{0}, \ldots, x_{k}\right), k \geq 0$, partly because the conditional distribution leads to a nonlinear filter that can be hard to implement, and partly because we seek for a sequence of gains $G(k)$ that can be pre-computed offline. The corresponding control problem is then defined as

$$
J_{N}^{*}\left(x_{0}, \pi_{0}\right)=\min _{u_{0}, \ldots, u_{N-1}} J_{N}\left(x_{0}, \pi_{0}\right) \quad \text { s.t. (1) and (3). }
$$

The main contribution of this paper is twofold. First, it presents a numerical method that computes the necessary optimal condition for the control problem posed in (4). The method in based on a monotone strategy, iterated at each step by a variational approach, that produces the convergence to a set of gain matrices $\mathbf{G}=\{G(0), \ldots, G(N-1)\}$ that satisfies the optimality condition, see Theorem 3.2. The second contribution is the application of the gain sequence $\mathbf{G}$ to control the speed of a real DC Motor device subject to abrupt power failures. The laboratory device is adapted to suffer power failures according to a homogeneous Markov chain. These elements constitute the main novelty of this paper.

The paper is organized as follows. In Section 2, we introduce the notation and problem formulation. In Section 3, we present a necessary condition for optimality and a method to compute this optimal condition. In Section 4, we deal with a practical application of the derived results in a DC Motor device. Finally, Section 5 presents concluding remarks. 


\section{DEFINITIONS AND BASIC CONCEPTS}

Let $\mathcal{R}^{r}$ denote the usual $r$-th dimensional Euclidean space, and let $\mathcal{M}^{r, s}\left(\mathcal{M}^{r}\right)$ represent the linear space formed by all $r \times s(r \times r)$ real matrices. Let $\mathcal{S}^{r}$ represent the normed linear subspace of $\mathcal{M}^{r}$ of symmetric matrices such as $\left\{U \in \mathcal{M}^{r}: U=U^{\prime}\right\}$, where $U^{\prime}$ denotes the transpose of $U$. Consider also $\mathcal{S}^{r 0}\left(\mathcal{S}^{r+}\right)$ its closed (open) convex cone of positive semidefinite (definite) matrices $\left\{U \in \mathcal{S}^{r}: U \geq 0(>0)\right\}$. Let $\mathscr{S}:=\{1, \ldots, \sigma\}$ be a finite set, and let $\mathbb{M}^{r, s}$ denote the linear space formed by a number $\sigma$ of matrices such that $\mathbb{M}^{r, s}=\left\{U=\left(U_{1}, \ldots, U_{\sigma}\right): U_{i} \in \mathcal{M}^{r, s}, i \in \mathscr{S}\right\}$; also $\mathbb{M}^{r} \equiv \mathbb{M}^{r, r}$. Moreover, we set $\mathbb{S}^{r}=\left\{U=\left(U_{1}, \ldots, U_{\sigma}\right): U_{i} \in \mathcal{S}^{r}, i \in \mathscr{S}\right\}$, and we write $\mathbb{S}^{r 0}\left(\mathbb{S}^{r+}\right)$ when $U_{i} \in \mathcal{S}^{r 0}\left(\in \mathcal{S}^{r+}\right)$ for all $i \in \mathscr{S}$.

We employ the ordering $U>V(U \geq V)$ for elements of $\mathbb{S}^{r}$, meaning that $U_{i}-V_{i}$ is positive definite (semi-definite) for all $i \in \mathscr{S}$, and similarly for other mathematical relations. Let $\operatorname{tr}\{\cdot\}$ be the trace operator. When applied for some $U \in \mathbb{S}^{n}$, the operator $\operatorname{tr}\{U\}$ signifies $\left(\operatorname{tr}\left\{U_{1}\right\}, \ldots, \operatorname{tr}\left\{U_{\sigma}\right\}\right)$. Define the inner product on the space $\mathbb{M}^{r, s}$ as

$$
\langle U, V\rangle=\sum_{i=1}^{\sigma} \operatorname{tr}\left\{U_{i}^{\prime} V_{i}\right\}, \quad \forall V, U \in \mathbb{M}^{r, s},
$$

and the Frobenius norm $\|U\|_{2}^{2}=\langle U, U\rangle$.

The transition probability matrix is denoted by $\mathbb{P}=\left[p_{i j}\right]$, for all $i, j \in \mathscr{S}$. The state of the Markov chain at a certain time $k$ is determined according to an associated probability distribution $\pi(k)$ on $\mathscr{S}$, namely, $\pi_{i}(k):=\operatorname{Pr}\left(\theta_{k}=i\right)$. Considering the column vector $\pi(k)=\left[\pi_{1}(k), \ldots, \pi_{\sigma}(k)\right]^{\prime}$, the state distribution of the chain, $\pi(k)$, is defined as $\pi(k)=\left(\mathbb{P}^{\prime}\right)^{k} \pi(0)$. Given $U \in \mathbb{M}^{r, s}$ and $\pi(k) \in \mathcal{R}^{\sigma}$, $k \geq 0$, we let $\pi(k) U$ represent the operation $\left(\pi_{1}(k) U_{1}, \ldots, \pi_{\sigma}(k) U_{\sigma}\right)$.

Associated with the system (1)-(2), we define $A \in \mathbb{M}^{r}, B \in \mathbb{M}^{r, s}, H \in \mathbb{M}^{r, q}, Q \in \mathbb{S}^{r 0}, R \in \mathbb{S}^{s+}$ and $F \in \mathbb{S}^{r 0}$. In addition, we define the operators $\mathscr{D}=\left\{\mathscr{D}_{i}, i \in \mathscr{S}\right\}: \mathbb{S}^{n 0} \mapsto \mathbb{S}^{n 0}$ and $\mathscr{E}=\left\{\mathscr{E}_{i}, i \in\right.$ $\mathscr{S}\}: \mathbb{S}^{n 0} \mapsto \mathbb{S}^{n 0}$, respectively, as

$$
\mathscr{D}_{i}(U):=\sum_{j=1}^{\sigma} p_{j i} U_{j}, \quad \mathscr{E}_{i}(U):=\sum_{j=1}^{\sigma} p_{i j} U_{j}, \quad \forall i \in \mathscr{S}, \forall U \in \mathbb{S}^{n 0}
$$

The class of all admissible gain sequences $\mathbf{G}=\{G(0), \ldots, G(N-1)\}$ as in (3) is represented by $\mathscr{G}$. Note that the corresponding closed-loop matrix sequence $A(k) \in \mathbb{M}^{r}$ satisfies

$$
A_{i}(k):=A_{i}+B_{i} G(k), \quad \forall i \in \mathscr{S}, k=0, \ldots, N-1 .
$$

Let us define the conditional second moment matrix of the system state $x_{k}, k \geq 0$, as

$$
X_{i}(k)=\mathrm{E}\left[x_{k} x_{k}^{\prime} \mathbb{1}_{\left\{\theta_{k}=i\right\}}\right], \quad \forall i \in \mathscr{S}, \forall k \geq 0,
$$

where $\mathbb{1}_{\{\cdot\}}$ stands for the Dirac measure. Setting $X(k)=\left\{X_{1}(k), \ldots, X_{\sigma}(k)\right\} \in \mathbb{S}^{n 0}$ for every $k \geq 0$, we obtain the recurrence [7, Prop. 3.35]

$$
X(k+1)=\mathscr{D}\left(A(k) X(k) A(k)^{\prime}+\pi(k) H H^{\prime}\right), \quad \forall k \geq 0,
$$

with $X_{i}(0)=\pi_{i}(0) x_{0} x_{0}^{\prime}$ for each $i \in \mathscr{S}$. In addition, let us define the sets $L(k) \in \mathbb{S}^{r 0}$ and $\omega(k) \in$ $\mathbb{S}^{10}, k=0, \ldots, N$, from the coupled recurrence equations

$$
L_{i}(k)=Q_{i}+G(k)^{\prime} R_{i} G(k)+A_{i}(k)^{\prime} \mathscr{E}_{i}(L(k+1)) A_{i}(k), \quad L_{i}(N)=F_{i}, \forall i \in \mathscr{S}
$$

and

$$
\omega_{i}(k)=\mathscr{E}_{i}(\omega(k+1))+\operatorname{tr}\left\{\mathscr{E}_{i}(L(k+1)) H_{i} H_{i}^{\prime}\right\}, \quad \omega_{i}(N)=0, \quad \forall i \in \mathscr{S} .
$$

The proof of the next result is given in Appendix. 
Lemma 2.1

Given $\mathbf{G} \in \mathscr{G}$, the next set of identities hold for each $k=0, \ldots, N-1$ :

$$
\begin{aligned}
& \mathrm{E}_{x_{0}, \pi_{0}}\left[\sum_{\ell=k}^{N-1} x_{\ell}^{\prime}\left(Q_{\theta_{\ell}}+G(\ell)^{\prime} R_{\theta_{\ell}} G(\ell)\right) x_{\ell}+x_{N}^{\prime} F_{\theta_{N}} x_{N}\right] \\
& =\sum_{\ell=k}^{N-1} \sum_{i=1}^{\sigma} \operatorname{tr}\left\{\left(Q_{i}+G(\ell)^{\prime} R_{i} G(\ell)\right) X_{i}(\ell)+F_{i} X_{i}(N)\right\}=\langle L(k), X(k)\rangle+\pi(k)^{\prime} \omega(k) .
\end{aligned}
$$

In view of the identity in (10), we have

$$
J_{N}\left(x_{0}, \theta_{0}\right)=\langle L(0), X(0)\rangle+\pi(0)^{\prime} \omega(0) .
$$

Thus, the stochastic control problem posed in (4) can be recast as that of minimizing the deterministic functional in (11) with respect to the gain sequence $\mathbf{G} \in \mathscr{G}$. This fact lead us to focus the remaining analysis on the deterministic expression of (11).

\section{MAIN RESULTS}

For sake of clarity, let us represent by $J_{\mathbf{G}}$ the cost $J_{N}\left(x_{0}, \theta_{0}\right)$ when evaluated for $\mathbf{G} \in \mathscr{G}$. The next result presents the necessary optimal condition for the considered control problem.

Theorem 3.1 (Necessary optimal condition)

Suppose that $\mathbf{G}=\{G(0), \ldots, G(N-1)\} \in \mathscr{G}$ is such that $J_{\mathbf{G}}=\min _{\mathbf{K} \in \mathscr{G}} J_{\mathbf{K}}$. Then, for each $k=0, \ldots, N-1$,

$$
\sum_{i=1}^{\sigma}\left[\left(R_{i}+B_{i}^{\prime} \mathscr{E}_{i}(L(k+1)) B_{i}\right) G(k)+B_{i}^{\prime} \mathscr{E}_{i}(L(k+1)) A_{i}\right] X_{i}(k)=0,
$$

where $X(k) \in \mathbb{S}^{r 0}$ and $L(k) \in \mathbb{S}^{r 0}$ are as in (7) and (8), respectively.

Proof

For some $k \geq 0$, let us assume that $G(0), \ldots, G(k-1), G(k+1), \ldots, G(N-1)$ are fixed optimal minimizers and $G(k)$ is a free design variable. In this case, both $X(0), \ldots, X(k)$ and $L(k+$ $1), \ldots, L(N)$ are fixed, and this allows us to deduce from the identity of (10) that

$$
\arg \min _{G(k)} J_{\mathbf{G}}=\arg \min _{G(k)}\langle L(k), X(k)\rangle+\pi(k)^{\prime} \omega(k) .
$$

Hence

$$
\begin{aligned}
& \arg \min _{G(k)} J_{\mathbf{G}} \\
&=\arg \min _{G(k)}\left[\sum_{i=1}^{\sigma} \operatorname{tr}\left\{\left[Q_{i}+G(k)^{\prime} R_{i} G(k)+\left(A_{i}+B_{i} G(k)\right)^{\prime} \mathscr{E}_{i}(L(k+1))\left(A_{i}+B_{i} G(k)\right)\right] X_{i}(k)\right\}\right. \\
&\left.\quad+\sum_{i=1}^{\sigma} \pi_{i}(k)\left(\mathscr{E}_{i}(\omega(k+1))+\operatorname{tr}\left\{\mathscr{E}_{i}(L(k+1)) H_{i} H_{i}^{\prime}\right\}\right)\right] .
\end{aligned}
$$

Taking the differentiation with respect to $G(k)$ in the expression within the last brackets, we obtain the expression in (12) and the proof is completed.

Remark 3.1

An interesting open question is whether the necessary optimal condition of Theorem 3.1 is also sufficient. Convexity can not be used to conclude sufficiency because the optimization approach is not convex at all, as the next example illustrates. 


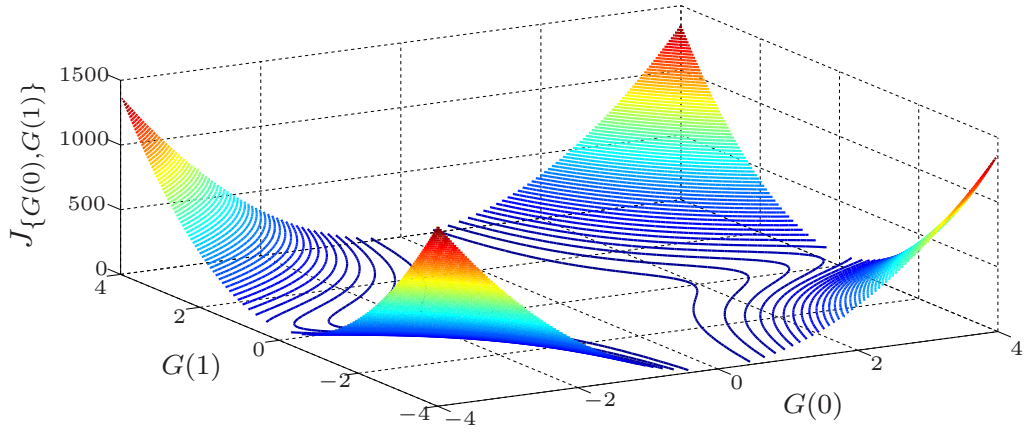

Figure 1. Three dimensional contour plot of the cost $J_{\{G(0), G(1)\}}$ as in Example 3.1.

Example 3.1

Consider the single-input single-output MJLS as in (1) with parameters $A_{1}=0.3, A_{2}=0.1, B_{1}=$ $-1, B_{2}=1, H_{i}=0, Q_{i}=0.4, R_{i}=1, F_{i}=0.5, i=1,2, N=2, x(0)=2$, and $\mu_{0}=\left[\begin{array}{ll}0.25 & 0.75\end{array}\right]$. We consider the stochastic matrix $\mathbb{P}=\left[p_{i j}\right], i, j=1,2$ as $p_{11}=0.6, p_{12}=0.4, p_{12}=0.2$, and $p_{22}=0.8$. After some algebraic manipulations on (11), one can rewrite the cost equivalently as

$$
\begin{aligned}
J_{\{G(0), G(1)\}=} & 1.6+4 G(0)^{2}+\left(0.4+G(1)^{2}\right)(0.3-G(0))^{2}+\left(1.2+3 G(1)^{2}\right)(0.1+G(0))^{2} \\
& +0.3(0.3-G(1))^{2}(0.3-G(0))^{2}+0.2(0.1+G(1))^{2}(0.3-G(0))^{2} \\
& +0.1(0.3-G(1))^{2}(0.1+G(0))^{2}+0.4(0.1+G(1))^{2}(0.1+G(0))^{2} .
\end{aligned}
$$

The functional in (13) is not convex as one can inspect in the contour plot of Fig 1. Note also in the figure that the function has a unique minimum with multiple solutions.

Remark 3.2

It should be noted that the coupled equations (7), (8), and (12) are nonlinear with respect to $\mathbf{G}=\{G(0), \ldots, G(N-1)\} \in \mathscr{G}$, and their evaluation represents a challenge for analytical and numerical fronts. The method of the next section represents a contribution towards this direction since it computes $\mathbf{G} \in \mathscr{G}$ that satisfies simultaneously (7), (8), and (12).

\subsection{Numerical method for the necessary optimal condition}

In this section we provide a method for evaluating the necessary optimal condition of Theorem 3.1. The idea of the method is to employ a variational principle to produce monotone cost functions. On the convergence, it provides a gain sequence $\mathbf{G} \in \mathscr{G}$ that satisfies the optimality condition of Theorem 3.1.

To present the method, we require some additional notation. Let $\eta=0,1, \ldots$ be an iteration index. For some given sequence

$$
\mathbf{G}[\eta]:=\left\{G^{[\eta]}(0), \ldots, G^{[\eta]}(N-1)\right\} \in \mathscr{G}, \eta=0,1, \ldots
$$

let us define

$$
A_{i}^{[\eta]}(k):=A_{i}+B_{i} G^{[\eta]}(k), \quad Q_{i}^{[\eta]}(k):=Q_{i}+G^{[\eta]}(k)^{\prime} R_{i} G^{[\eta]}(k), \quad \forall i \in \mathscr{S}, k=0, \ldots, N-1 .
$$

Let us now consider the following algorithm.

- Step 1: Set the iterations counter $\eta=0$. Pick an arbitrary initial sequence $\mathbf{G}[0] \in \mathscr{G}$.

- Step 2: For each $k=1, \ldots, N$, find $X^{[\eta]}(k) \in \mathbb{S}^{r 0}$, the solution of the following set of equations:

$$
X^{[\eta]}(k)=\mathscr{D}\left(A^{[\eta]}(k-1) X^{[\eta]}(k-1) A^{[\eta]}(k-1)^{\prime}+\pi(k-1) H H^{\prime}\right),
$$

with $X^{[\eta]}(0)=X(0)$. Set $\eta=\eta+1$ and go to Step 3 . 
- Step 3: Set $L^{[\eta]}(N)=F$ and $k=N-1$. Let $G^{[\eta]}(k) \in \mathcal{M}^{s, r}$ be defined by

$$
\sum_{i=1}^{\eta}\left[\left(R_{i}+B_{i}^{\prime} \mathscr{E}_{i}\left(L^{[\eta]}(k+1)\right) B_{i}\right) G^{[\eta]}(k)+B_{i}^{\prime} \mathscr{E}_{i}\left(L^{[\eta]}(k+1)\right) A_{i}\right] X_{i}^{[\eta-1]}(k)=0 .
$$

Evaluate the expression

$$
\left(G^{[\eta]}(k)-G^{[\eta-1]}(k)\right) X_{i}^{[\eta-1]}(k)=0
$$

and set $G^{[\eta]}(k)=G^{[\eta-1]}(k)$ only if (15) holds true for all $i \in \mathscr{S}$. Compute $L^{[\eta]}(k) \in \mathbb{S}^{r 0}$ and $\omega^{[\eta]}(k) \in \mathbb{S}^{1}$ from the recurrence

$$
\begin{array}{ll}
L^{[\eta]}(k)=Q^{[\eta]}(k)+A^{[\eta]}(k)^{\prime} \mathscr{E}\left(L^{[\eta]}(k+1)\right) A^{[\eta]}(k), & L^{[\eta]}(N)=F, \\
\omega^{[\eta]}(k)=\mathscr{E}\left(\omega^{[\eta]}(k+1)\right)+\operatorname{tr}\left\{\mathscr{E}\left(L^{[\eta]}(k+1)\right) H H^{\prime}\right\}, & \omega^{[\eta]}(N)=0 .
\end{array}
$$

Set $k=k-1$; if $k \geq 0$ then return to the beginning of Step 3 .

- Step 4: Compute the cost $J_{\mathbf{G}[\eta]}=\left\langle L^{[\eta]}(0), X(0)\right\rangle+\pi(0)^{\prime} \omega^{[\eta]}(0)$. If the evaluation of the difference $J_{\mathbf{G}[\eta-1]}-J_{\mathbf{G}[\eta]}$ is sufficiently small, then stop the algorithm. Otherwise, return to the beginning of Step 2.

Remark 3.3

The equation (14) can be transformed into a system of linear equations of the form $\mathcal{A} w=b$, whose solution can be obtained to a desired precision using efficient numerical methods available in literature. In fact, by applying the Kronecker product and the column stacking operator (denoted by $\otimes$ and vec respectively) one obtains $w=\operatorname{vec}\left(G^{[\eta]}(k)\right), \mathcal{A}=\sum_{i=1}^{\eta}\left[X_{i}^{[\eta-1]}(k) \otimes\right.$ $\left.\left(R_{i}+B_{i}^{\prime} \mathscr{E}_{i}\left(L^{[\eta]}(k+1)\right) B_{i}\right)\right]$ and $b=-\operatorname{vec}\left[\sum_{i=1}^{\eta}\left[B_{i}^{\prime} \mathscr{E}_{i}\left(L^{[\eta]}(k+1)\right) A_{i}\right] X_{i}^{[\eta-1]}(k)=0\right]$.

Remark 3.4

The algorithm can be implemented in a receding horizon framework. At each time instant $\ell \geq 0$, the gain sequence $G(t), \ell \leq t \leq \ell+N-1$ is calculated and only $G(t), t=\ell$ is implemented. In order to compute this gain sequence, assuming $X(\ell)=M$ with $M$ given, one can employ the algorithm with a time displacement $k=t-\ell$ : set $X(0)=M$ and obtain the gain sequence $G(k)$, $0 \leq k \leq N-1$ as indicated in the algorithm (producing $G(t), \ell \leq t \leq \ell+N-1$ ). Note that only the covariance matrix $X(\ell)=M$ (or an estimate of it) is required at time instant $\ell$ to obtain the corresponding receding horizon gain.

\section{Theorem 3.2}

The gain sequences $\mathbf{G}[\eta] \in \mathscr{G}, \eta=0,1, \ldots$, generated in the algorithm of Steps $1-4$, satisfy the monotone property $J_{\mathbf{G}[\eta]} \geq J_{\mathbf{G}[\eta+1]}$. Moreover, the limit $\mathbf{G}=\lim _{\eta \rightarrow \infty} \mathbf{G}[\eta]$ exists and it satisfies the necessary optimal condition of Theorem 3.1.

Proof

We divide the proof of Theorem 3.2 into two parts. The first part introduces an evaluation for the cost corresponding to two different gain sequences, and the second one proves that the cost corresponding to the gain sequences from Step 3 is monotonically non-increasing. As a byproduct, we get that the gain sequences converge to a sequence that satisfies the optimality condition of Theorem 3.1.

To begin with, we need to introduce some additional notation. For a given gain sequence $\mathbf{G}=\{G(0), \ldots, G(N-1)\} \in \mathscr{G}$, let us consider the operator

$$
\mathscr{L}_{i, \mathbf{G}}^{k}(U):=\left(A_{i}+B_{i} G(k)\right)^{\prime} \mathscr{E}_{i}(U)\left(A_{i}+B_{i} G(k)\right), \quad k=0, \ldots, N-1, \forall i \in \mathscr{S}, U \in \mathbb{S}^{r},
$$

so that we can write

$$
L_{i, \mathbf{G}}(k)=Q_{i}+G(k)^{\prime} R_{i} G(k)+\mathscr{L}_{i, \mathbf{G}}^{k}\left(L_{\mathbf{G}}(k+1)\right), \quad k=0, \ldots, N-1, \forall i \in \mathscr{S},
$$


with $L_{\mathrm{G}}(N)=F$.

After some algebraic manipulation (see Appendix for a detailed proof), we have

$$
L_{\mathbf{G}}(k)-L_{\mathbf{K}}(k)=\delta_{\mathbf{G}, \mathbf{K}}^{k}+\mathscr{L}_{\mathbf{K}}^{k}\left(L_{\mathbf{G}}(k+1)-L_{\mathbf{K}}(k+1)\right), \quad k=0, \ldots, N-1,
$$

with both $\mathbf{G}$ and $\mathbf{K}$ belonging to $\mathscr{G}$, where

$$
\delta_{i, \mathbf{G}, \mathbf{K}}^{k}:=\left(G(k)-Z_{i}^{k}\right)^{\prime} \Lambda_{i, \mathbf{G}}^{k+1}\left(G(k)-Z_{i}^{k}\right)-\left(K(k)-Z_{i}^{k}\right)^{\prime} \Lambda_{i, \mathbf{G}}^{k+1}\left(K(k)-Z_{i}^{k}\right), \forall i \in \mathscr{S},
$$

with $\Lambda_{i, \mathbf{G}}^{k}:=R_{i}+B_{i}^{\prime} \mathscr{E}_{i}\left(L_{\mathbf{G}}(k)\right) B_{i}$ and $Z_{i}^{k}:=-\left(\Lambda_{i, \mathbf{G}}^{k+1}\right)^{-1} B_{i}^{\prime} \mathscr{E}_{i}\left(L_{\mathbf{G}}(k+1)\right) A_{i}$. Moreover, if $\mathbf{G}=$ $\mathbf{G}[\eta] \in \mathscr{G}$ is the gain sequence that satisfies (14) and $X(k)=X^{[\eta-1]}(k), k=0, \ldots, N$, is the corresponding second moment trajectory from Step 2, then we have [9, p. 1123]

$$
\begin{aligned}
\left\langle X(k), \delta_{\mathbf{G}, \mathbf{K}}^{k}\right\rangle & =\left\|\left(\Lambda_{\mathbf{G}}^{k+1}\right)^{\frac{1}{2}}\left(G(k)-Z^{k}\right) X(k)^{\frac{1}{2}}\right\|_{2}^{2}-\left\|\left(\Lambda_{\mathbf{G}}^{k+1}\right)^{\frac{1}{2}}\left(K(k)-Z^{k}\right) X(k)^{\frac{1}{2}}\right\|_{2}^{2} \\
& =-\left\|\left(\Lambda_{\mathbf{G}}^{k+1}\right)^{\frac{1}{2}}(G(k)-K(k)) X(k)^{\frac{1}{2}}\right\|_{2}^{2} .
\end{aligned}
$$

The expression of (20) will be useful on evaluating the quantity $J_{\mathbf{G}[\eta]}-J_{\mathrm{G}[\eta-1]}$. Indeed, we derive in the sequel the arguments to show that

$$
J_{\mathbf{G}[\eta]}-J_{\mathbf{G}[\eta-1]}=\left\langle X(k), \delta_{\mathbf{G}}^{k}\right\rangle=-\left\|\left(\Lambda_{\mathbf{G}}^{k+1}\right)^{\frac{1}{2}}(G(k)-K(k)) X(k)^{\frac{1}{2}}\right\|_{2}^{2} .
$$

This result is important because it enables us to conclude that the cost sequence generated by Steps $1-4$ is monotone, i.e., there holds $J_{\mathbf{G}[\eta-1]} \geq J_{\mathbf{G}[\eta]}$ for every $\eta=1,2, \ldots$, thus showing the first statement of Theorem 3.2.

To show the identity in (21), let us define the sequences

$$
\mathcal{G}^{[\eta, k]}:=\left\{G^{[\eta-1]}(0), \ldots, G^{[\eta-1]}(k-1), G^{[\eta]}(k), \ldots, G^{[\eta]}(N)\right\},
$$

for each $k=0 \ldots, N$, and set $\mathcal{G}^{[\eta]}=\mathcal{G}^{[\eta, 0]}$. Recall the expression of the cost in (10), and note that the last element of $\mathcal{G}^{[\eta, k]}$, i.e., $G^{[\eta]}(N)$, does not influence the value of the cost, so that $J_{\mathcal{G}[\eta-1]}=J_{\mathcal{G}[\eta, N]}$.

The Step 3 calculates $G^{[\eta]}(k)$ backwards in time. Thus, when an iteration of Step 3 occurs, the element $G^{[\eta-1]}(k)$ in $\mathcal{G}^{[\eta, k+1]}$ is modified to $G^{[\eta]}(k)$ in $\mathcal{G}^{[\eta, k]}$, while the other elements remain unchanged. This observation leads to

$$
m>k \Rightarrow L_{\mathcal{G}[\eta, k]}(m)=L_{\mathcal{G}[\eta, k+1]}(m) .
$$

Let us now define the recurrence

$$
\omega_{i, \mathbf{G}}(k)=\mathscr{E}_{i}\left(\omega_{\mathbf{G}}(k+1)\right)+\operatorname{tr}\left\{\mathscr{E}_{i}\left(L_{\mathbf{G}}(k+1)\right) H_{i} H_{i}^{\prime}\right\}, \quad \omega_{i, \mathbf{G}}(N)=0, \quad \forall i \in \mathscr{S} .
$$

It follows from (24) that

$$
\begin{aligned}
& \omega_{\mathcal{G}[\eta, k]}(k)-\omega_{\mathcal{G}[\eta, k+1]}(k)=\mathscr{E}\left(\omega_{\mathcal{G}[\eta, k]}(k+1)-\omega_{\mathcal{G}[\eta, k+1]}(k+1)\right) \\
& +\operatorname{tr}\left\{\mathscr{E}\left(L_{\mathcal{G}[\eta, k]}(k+1)-L_{\mathcal{G}[\eta, k+1]}(k+1)\right) H H^{\prime}\right\}
\end{aligned}
$$

with $\omega_{\mathcal{G}[\eta, k]}(N)=\omega_{\mathcal{G}}^{[\eta, k+1]}(N)=0$. Since the rightmost term of (25) is null due to the identity in (23), we can apply a simple induction argument on the resulting expression from (25) to conclude that

$$
\omega_{\mathcal{G}^{[\eta, k]}}(k)=\omega_{\mathcal{G}^{[\eta, k+1]}}(k), \quad k=0, \ldots, N-1 .
$$

One can employ a similar reasoning for the recurrence in (7) to show that

$$
k \geq m \geq 0 \Rightarrow X_{\mathcal{G}}^{[\eta, k]}(m)=X_{\mathcal{G}[\eta, k+1]}(m) .
$$

In particular, we can observe from Step 2 the validity of the identity

$$
X_{\mathcal{G}}{ }^{[\eta, k]}(k)=X^{[\eta-1]}(k), \quad k=0, \ldots, N .
$$


Now, we are able to prove the identity in (21). Indeed, from (10), we have

$$
\begin{aligned}
J_{\mathcal{G}[\eta, k]}-J_{\mathcal{G}^{[\eta, k+1]}}= & \left\langle X_{\mathcal{G}^{[\eta, k]}}(k), L_{\mathcal{G}^{[\eta, k]}}(k)\right\rangle+\pi(k)^{\prime} \omega_{\mathcal{G}}[\eta, k] \\
& -\left\langle X_{\mathcal{G}[\eta, k+1]}(k), L_{\mathcal{G}}[\eta, k+1]\right. \\
& (k)\rangle-\pi(k)^{\prime} \omega_{\mathcal{G}[\eta, k+1]}(k) .
\end{aligned}
$$

Now substituting (26)-(28) into (29), we get

$$
J_{\mathcal{G}[\eta, k]}-J_{\mathcal{G}[\eta, k+1]}=\left\langle X^{[\eta-1]}(k), L_{\mathcal{G}[\eta, k]}(k)-L_{\mathcal{G}[\eta, k+1]}(k)\right\rangle,
$$

or equivalently, we can invoke the identity of (18) to obtain

$$
J_{\mathcal{G}}^{[\eta, k]}-J_{\mathcal{G}}^{[\eta, k+1]}=\left\langle X^{[\eta-1]}(k), \delta_{\mathcal{G}^{[\eta, k], \mathcal{G}}[\eta, k+1]}^{k}+\mathscr{L}_{\mathcal{G}^{[\eta, k+1]}}^{k}\left(L_{\mathcal{G}^{[\eta, k]}}(k+1)-L_{\mathcal{G}^{[\eta, k+1]}}(k+1)\right)\right\rangle .
$$

But then we can employ (23) with $m=k+1$ to conclude that

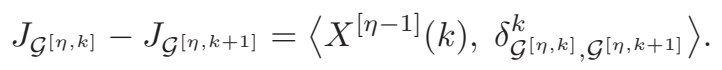

Hence, if we let

$$
\xi(k):=\left\|\left(\Lambda_{\mathcal{G}[\eta, k]}^{k+1}\right)^{\frac{1}{2}}\left(G^{[\eta]}(k)-G^{[\eta-1]}(k)\right) X^{[\eta-1]}(k)^{\frac{1}{2}}\right\|_{2}^{2}, \quad k=0, \ldots, N,
$$

then we can combine (20) and (30) to write

$$
J_{\mathcal{G}[\eta, k]}-J_{\mathcal{G}^{[\eta, k+1]}}=-\xi(k), \quad k=0, \ldots, N .
$$

Since the matrix $\Lambda_{i, \mathcal{G}[\eta, k]}^{k+1}$ is positive definite for each $i \in \mathscr{S}$, we have that $\xi(k)=0$ if and only if $\left(G^{[\eta]}(k)-G^{[\eta-1]}(k)\right) X_{i}^{[\eta-1]}(k)=0$ for all $i \in \mathscr{S}$. In this case, Step 3 assures that $G^{[\eta]}(k)=$ $G^{[\eta-1]}(k)$, which in turn implies that $\xi(k)=0$ if and only if $G^{[\eta]}(k)=G^{[\eta-1]}(k)$.

Finally, the result of Theorem 3.2 then follows by summing up (30) with respect to $k$, i.e.,

$$
J_{\mathcal{G}^{[\eta]}}-J_{\mathcal{G}^{[\eta-1]}}=\sum_{k=0}^{N-1}\left(J_{\mathcal{G}^{[\eta, k]}}-J_{\mathcal{G}^{[\eta, k+1]}}\right)=-\sum_{k=0}^{N-1} \xi(k) \leq 0,
$$

which shows the monotone non-increasing property of the cost sequence $J_{\mathcal{G}[\eta]}, \eta=0,1, \ldots$ As a byproduct, we have that $J_{\mathcal{G}^{[\eta-1]}}>J_{\mathcal{G}^{[\eta]}}$ whenever $G^{[\eta]}(k) \neq G^{[\eta-1]}(k)$, so that the limit $\lim _{\eta \rightarrow \infty} G^{[\eta]}(k)$ exists for every $k=0, \ldots, N-1$. This argument completes the proof of Theorem 3.2 .

\section{Remark 3.5}

The results of this paper can be quite easily extended to the scenario of clustered observation of the Markov state [10], in which one observes the variable $\psi_{k}$ taking values in the set $\mathscr{S}=\{1, \ldots, \sigma\}$ and satisfying $\psi_{k}=i$ whenever $\theta_{k} \in \mathscr{S}_{i}$, where $\mathscr{S}_{i}, 0 \leq i \leq \sigma$, forms a partition of $\mathscr{S}$. For example, if $\mathscr{S}=\{1, \ldots, 4\}, \mathscr{S}_{1}=\{1\}$ and $\mathscr{S}_{2}=\{2, \ldots, 4\}$, then $\bar{\psi}_{k}=2$ means that $\theta_{k} \in\{2, \ldots, 4\}$. Note that, if we define the function

$$
\psi(i)=\sum_{j=1}^{\sigma} j \mathbb{1}_{\left\{i \in \mathscr{S}_{j}\right\}},
$$

then $\psi_{k}=\psi(\theta(k))$ a.s.. We assume that the controller is in the form $u_{k}=G\left(k, \psi\left(\theta_{k}\right)\right) x_{k}$, hence the closed loop structure is now given by $A_{i}(k):=A_{i}+B_{i} G(k, \psi(i))$, for all $i \in \mathscr{S}$ and $k=$ $0, \ldots, N-1$, and the necessary condition for optimality reads as

$$
\sum_{i=1}^{\sigma}\left[\left(R_{i}+B_{i}^{\prime} \mathscr{E}_{i}(L(k+1)) B_{i}\right) G(k, \psi(i))+B_{i}^{\prime} \mathscr{E}_{i}(L(k+1)) A_{i}\right] X_{i}(k)=0 .
$$

The algorithm is altered accordingly by substituting (14) by (31). 


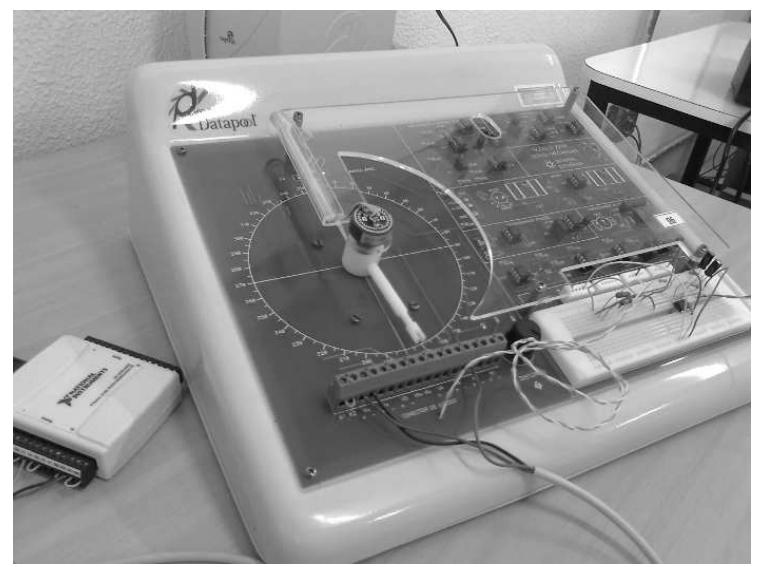

Figure 2. Laboratory DC Motor testbed used in the experiments of Section 4.

One interesting feature of (31) is that, assuming the cardinality of $\mathscr{S}_{j}$ is one for some $0 \leq j \leq \sigma$, that is, $\mathscr{S}_{j}=\{r\}$ for some $0 \leq r \leq \sigma$, then $\psi(\ell)=j$ only when $\ell=r$, allowing to obtain from (31) an analytical expression for $G(k, \psi(r))$

$$
G(k, \psi(r))=-\left(R_{r}+B_{r}^{\prime} \mathscr{E}_{r}(L(k+1)) B_{r}\right)^{-1} B_{r}^{\prime} \mathscr{E}_{r}(L(k+1)) A_{r},
$$

irrespectively of $X(k)$ and the other gains $G(k, \psi(i))$ in (31). In one extreme, the case when the mode is observed (complete observation) can be retrieved by setting $\mathscr{S}_{j}=\{j\}, 0 \leq j \leq$ $\sigma$. In this situation, $\psi(i)=i$ and the optimal gain is given by $G(k, i)=-\left(R_{i}+B_{i}^{\prime} \mathscr{E}_{i}(L(k+\right.$ 1)) $\left.B_{i}\right)^{-1} B_{i}^{\prime} \mathscr{E}_{i}(L(k+1)) A_{i}$. The algorithm converges in one iteration, and (16) is now equivalent to the well known Riccati difference equation for the jump linear quadratic problem [7, Ch. 4]. This also serves as an illustration that the dependence of the gains on the second moment matrices $X(k)$ in (31) is not a drawback of the methodology in this paper, it is a feature of the considered partial observation problem. In the other extreme, (31) and (14) are equivalent if one sets $\mathscr{S}_{1}=\mathscr{S}$.

\section{EXPERIMENTAL RESULTS FROM A DC MOTOR DEVICE}

This section presents a real implementation of the underlying Markovian controller for a DC Motor device. In practical terms, we use the results of the previous section to derive a strategy to control the speed of a real DC Motor device subject to abrupt failures. The equipment is altered to take these failures into account according to a prescribed Markov chain.

The experimental testbed is based on the DC Motor Module 2208, made up by Datapool Eletronica Ltda, Brazil, using a National Instruments USB-6008 data acquisition card to perform a physical link with the computer, see Fig. 2. The computer calls Matlab software to implement physically the controller and it makes use of the gain sequence that was pre-computed offline from Theorem 3.2.

It is known that the dynamics of DC Motors can be satisfactorily represented by second order linear systems [18], [22], [23]. In this case, the two system state variables are the angular velocity of the motor shaft and the electrical current consumed by the motor, which are represented in this project respectively by $v_{k}$ and $i_{k}, k \geq 0$. In practice, to measure the angular velocity, we use the manufacturer-provided tachogenerator that produces voltage proportional to the speed of the shaft; and to measure the electric current, we introduce in series with the motor a simple circuit composed by a shunt resistor connected with a pre-amplifier signal stage. First-order analog filters are used in the circuit to reduce high-frequency noise from the experimental data. The experiments of this project are conducted with a sampling period of 15.6118 milliseconds.

Abrupt failures on the power transmitted to the shaft play an important role in the speed of motors, and this fact motivates us to adjust the apparatus in order to impose power failures therein. Namely, 
we force the DC motor device to run under three distinct operation modes, i.e., the normal, low, and medium power modes, and these switching modes are programmed to occur according to a homogeneous Markov chain.

Under this failure scenario, we aim to control the speed of the DC motor so as to track the constant input reference of one radian per second. As a byproduct, we can assure that the steady-state error vanishes to zero. In fact, to accomplish this goal in practice, we modify the PI compensator schematic suggested in [21, Sec. 10.7.3] to cope with the discrete-time MJLS. As a result, by setting the system state as $x_{k} \equiv\left[v_{k} i_{k} x_{3, k}\right]^{\prime}$ (where $x_{3, k}$ represents the integrative term written as a discrete sum), we are able to model the DC Motor device subject to failures as the following discrete-time Markov jump linear system:

$$
x_{k+1}=A_{\theta_{k}} x_{k}+B_{\theta_{k}} u_{k}+H_{\theta_{k}} w_{k}+\Gamma_{\theta_{k}} r_{k}, \quad k \geq 0,
$$

where the parameters

$$
A_{i}=\left[\begin{array}{ccc}
a_{11}^{(i)} & a_{12}^{(i)} & 0 \\
a_{21}^{(i)} & a_{22}^{(i)} & 0 \\
a_{31}^{(i)} & 0 & a_{33}^{(i)}
\end{array}\right], B_{i}=\left[\begin{array}{c}
b_{1}^{(i)} \\
b_{2}^{(i)} \\
0
\end{array}\right], \Gamma_{i}=\left[\begin{array}{c}
0 \\
0 \\
\gamma^{(i)}
\end{array}\right], H_{i}=h^{(i)}, \quad i=1,2,3 .
$$

are given in Table I and II. The sequence $\left\{w_{k}\right\}$ on $\mathcal{R}^{2}$ represents an i.i.d. noise sequence with zero mean and covariance matrix equal to the identity matrix, $\left\{r_{k}\right\}$ on $\mathcal{R}$ denotes the tracking reference signal, and $\left\{u_{k}\right\}$ on $\mathcal{R}$ stands for the controller.

The design objective of this project is to control the speed of the real DC Motor device when sudden power failures occur. The practical experiment in the laboratory testbed implements the controller in the linear state-feedback form

$$
u_{k}=G(k) x_{k}, \quad \forall k \geq 0 .
$$

In the control design, we set the model (32) and (33) with $r(k) \equiv 0$ to get a matrix gain sequence $\mathbf{G}=\{G(0), \ldots, G(N-1)\}$ from Theorem 3.2 satisfying the necessary optimal condition of Theorem 3.1. This strategy is purposeful to improve attenuation of the real input disturbances $\omega(\cdot)$ with fast transient response for tracking problems, see [1], [16], and [23] for further details regarding deterministic systems. As a consequence, these specifications can be taken into account in our practical experiments designed for the tracking reference $r_{k} \equiv 1$. Indeed, we will see in the sequence that $\mathbf{G}$ engenders an interesting tracking behavior for the speed of the DC Motor device when failures happen.

To perform the experiments, we set $N=1800, \pi_{0}=\left[\begin{array}{lll}1 & 0 & 0\end{array}\right]^{\prime}$,

$$
Q_{i}=\left[\begin{array}{ccc}
q_{11}^{(i)} & q_{12}^{(i)} & 0 \\
q_{12}^{(i)} & q_{22}^{(i)} & 0 \\
0 & 0 & q_{33}^{(i)}
\end{array}\right], \text { and } F_{i}=\mathbf{0}_{2 \times 2}, \quad i=1,2,3
$$

with values shown in Table III.

The task of defining precisely the value of the stochastic matrix $\mathbb{P}$ may be cumbersome in some circumstances $[19,20,28,29,30]$. In this project, however, we are able to define $\mathbb{P}$ precisely as

$$
\mathbb{P}=\left[\begin{array}{lll}
0.84 & 0.07 & 0.09 \\
0.24 & 0.75 & 0.01 \\
0.11 & 0.08 & 0.81
\end{array}\right]
$$

We can see in Fig. 3 the experimental and simulated data of the angular velocity and electric current for some realization of the Markovian process. Notice in the figure that the experimental and simulated data tend to overlap each other, which is a strong indication that the MJLS model (32)(33) provides a good representation of the DC Motor device subject to power failures. In addition, one can see that the DC Motor speed $v_{k}$ follows the tracking reference $r_{k} \equiv 1$ with success, even 
though the power failures tend to deviate it from its reference target. The figure also presents the states of the Markov chain with respect to the normal $\left(\theta_{k}=1\right)$, low $\left(\theta_{k}=2\right)$, and medium $\left(\theta_{k}=3\right)$ power modes associated with the evolution of the system trajectory.

To clarify the influence of abrupt power failures on the DC Motor device in practice, we carry out a Monte Carlo based experiment. The idea of the Monte Carlo experimentation is to operate the DC Motor device to work out one thousand distinct random experiments, and the corresponding outcome is then used to obtain the mean and standard deviation of both the angular velocity and electric current of the device, see Fig. 4 for a pictorial representation. It is noteworthy that even in the real scenario of failures, the designed controller is able to drive with success the mean value of the DC Motor speed to the tracking reference value of one radian per second. The experimental values of the standard deviation are bounded and this indicates that the stochastic system is stable, c.f. [7, Ch. 3], [26].

\section{CONCLUDING REMARKS}

This work presents a method to compute a gain matrix sequence that satisfies a necessary optimal condition for the control problem of MJLS with no mode observation. The necessary optimal condition of Theorem 3.1, which is nonlinear with respect to the optimizing variables, is evaluated by means of a variational method that converges to the solution, see Theorem 3.2 in connection. In Section 4, the derived control strategy satisfying the optimal condition is applied in practice to control the speed of a real DC Motor device subject to abrupt power failures. The contribution of this approach is reinforced by the Monte Carlo experiment, which shows that even in the case with sudden power failures, the proposed MJLS controller with no mode observation is able to control the speed of the DC Motor device.

Table I. Parameters of the discrete-time MJLS representing a real DC motor device as in Section 4.

\begin{tabular}{ccccccc}
\hline Parameters & $a_{11}^{(i)}$ & $a_{12}^{(i)}$ & $a_{21}^{(i)}$ & $a_{22}^{(i)}$ & $a_{31}^{(i)}$ & $a_{33}^{(i)}$ \\
\hline$i=1$ & -0.479908 & 5.1546 & -3.81625 & 14.4723 & 0.139933 & -0.925565 \\
\hline$i=2$ & -1.60261 & 9.1632 & -0.5918697 & 3.0317 & 0.0740594 & -0.43383 \\
\hline$i=3$ & 0.634617 & 0.917836 & -0.50569 & 2.48116 & 0.386579 & 0.0982194 \\
\hline
\end{tabular}

Table II. Parameters of the discrete-time MJLS representing a real DC motor device as in Section 4.

\begin{tabular}{ccccc}
\hline Parameters & $b_{1}^{(i)}$ & $b_{2}^{(i)}$ & $h^{(i)}$ & $\gamma^{(i)}$ \\
\hline$i=1$ & 5.87058212 & 15.50107 & 0.1 & 0.11762727 \\
\hline$i=2$ & 10.285129 & 2.2282663 & 0.1 & -0.1328741 \\
\hline$i=3$ & 0.7874647 & 1.5302844 & 1 & 0.1632125 \\
\hline
\end{tabular}

Table III. Parameters for the experimental testbed of Section 4.

\begin{tabular}{cccccc}
\hline Parameters & $q_{11}^{(i)}$ & $q_{12}^{(i)}$ & $q_{22}^{(i)}$ & $q_{33}^{(i)}$ & $R_{i}$ \\
\hline$i=1$ & 0.24 & 0.61 & 2.1 & 0.7 & 2 \\
\hline$i=2$ & 0.8 & -0.512 & 0.676 & 0.1 & 2 \\
\hline$i=3$ & 0 & 0 & 0 & 0 & 1000 \\
\hline
\end{tabular}



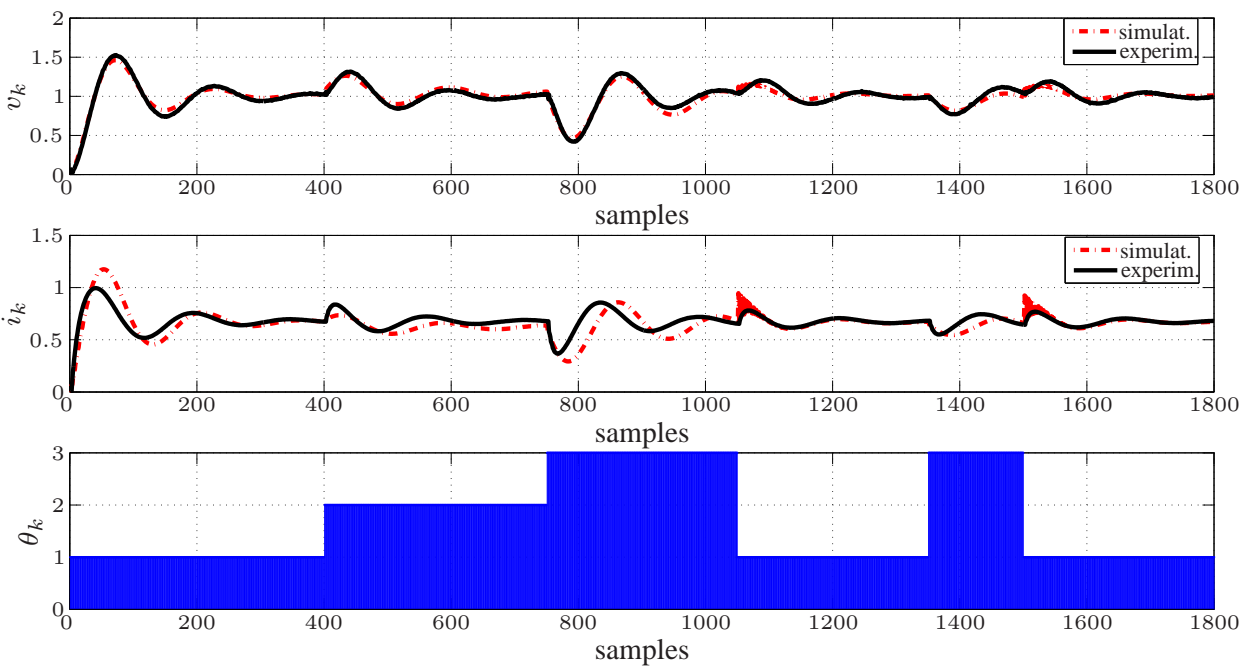

Figure 3. A sample-path of the angular velocity and electric current obtained from both real and simulated data using the MJLS control strategy of Theorem 3.2. The corresponding state of the Markov chain is depicted in the third picture.
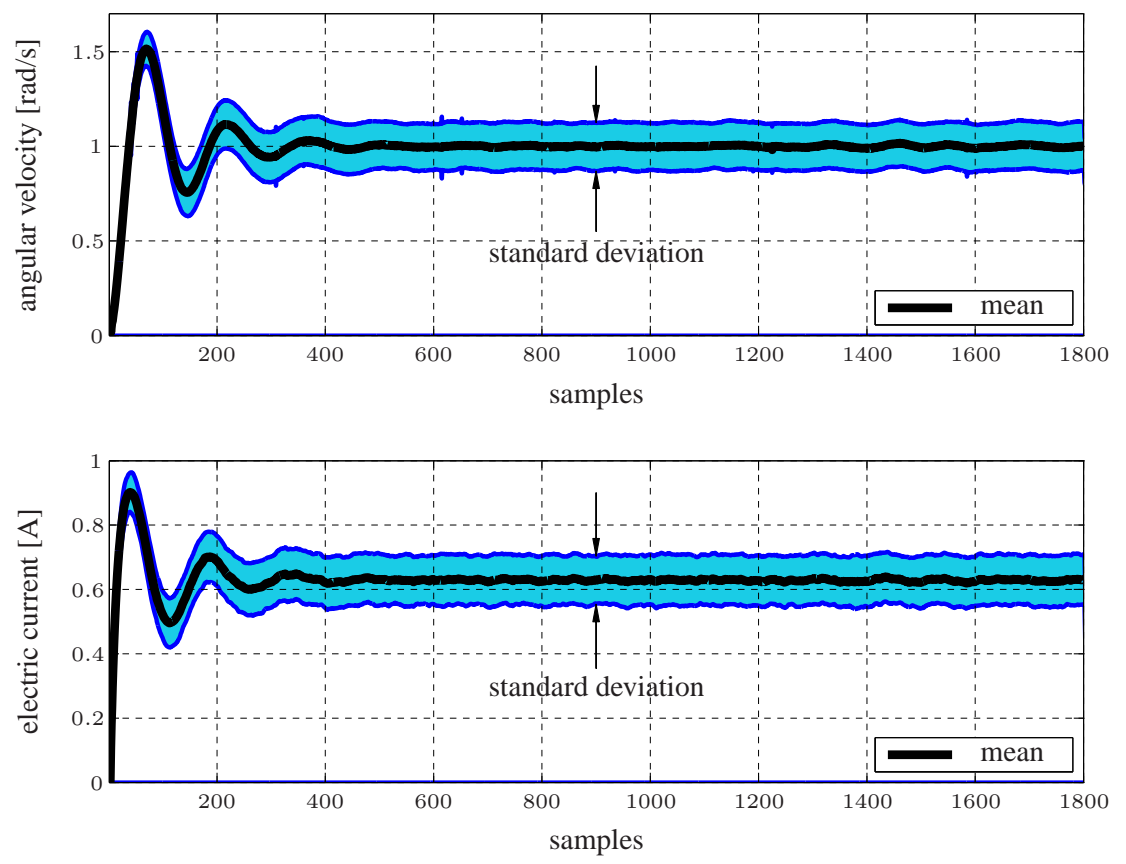

Figure 4. Mean and standard deviation values of the angular velocity and electric current obtained from Monte Carlo practical experiments. The MJLS control strategy of Theorem 3.2 is used to generate the corresponding experimental data.

\section{APPENDIX}

Proof

(Proof of Lemma 2.1). Given $\mathbf{G} \in \mathscr{G}$, let us define the random variable $W(t, \cdot)$ for each $t=0, \ldots, N$ 
as

$$
W\left(t, x_{t}, \theta_{t}\right)=\mathrm{E}\left[\sum_{\ell=t}^{N-1} x_{\ell}^{\prime}\left(Q_{\theta_{\ell}}+G(\ell)^{\prime} R_{\theta_{\ell}} G(\ell)\right) x_{\ell}+x_{N}^{\prime} F_{\theta_{N}} x_{N} \mid x_{t}, \theta_{t}\right],
$$

with terminal condition $W\left(N, x_{N}, \theta_{N}\right)=x_{N}^{\prime} F_{\theta_{N}} x_{N}$. Since the joint process $\left\{x_{t}, \theta_{t}\right\}$ is Markovian [7, p. 31], we can write the identity

$$
\begin{aligned}
& W\left(t, x_{t}, \theta_{t}\right)=x_{t}^{\prime}\left(Q_{\theta_{t}}+G(t)^{\prime} R_{\theta_{t}} G(t)\right) x_{t} \\
& +\mathrm{E}\left[\mathrm{E}\left[\sum_{\ell=t+1}^{N-1} x_{\ell}^{\prime}\left(Q_{\theta_{\ell}}+G(\ell)^{\prime} R_{\theta_{\ell}} G(\ell)\right) x_{\ell}+x_{N}^{\prime} F_{\theta_{N}} x_{N} \mid x_{t+1}, \theta_{t+1}\right] \mid x_{t}, \theta_{t}\right] \\
& \quad=x_{t}^{\prime}\left(Q_{\theta_{t}}+G(t)^{\prime} R_{\theta_{t}} G(t)\right) x_{t}+\mathrm{E}\left[W\left(t+1, x_{t+1}, \theta_{t+1}\right) \mid x_{t}, \theta_{t}\right] .
\end{aligned}
$$

Setting $x_{t}=x \in \mathcal{R}^{r}$ and $\theta_{t}=i \in \mathscr{S}$, we now show by induction that

$$
W(t, x, i)=x^{\prime} L_{i}(t) x+\omega_{i}(t),
$$

where $L(t) \in \mathbb{S}^{r 0}$ and $\omega(t) \in \mathbb{M}^{1}, t=0, \ldots, N$, satisty (8) and (9), respectively. Indeed, take $t=N$ and it is immediate that

$$
W\left(N, x_{N}, \theta_{N}\right)=x_{N}^{\prime} F_{\theta_{N}} x_{N}=x_{N}^{\prime} L_{\theta_{N}}(N) x_{N},
$$

which shows the result for $t=N$. Now, suppose that (36) holds for $t=m+1$, i.e., that

$$
W\left(m+1, x_{m+1}, \theta_{m+1}\right)=x_{m+1}^{\prime} L_{\theta_{m+1}}(m+1) x_{m+1}+\omega_{\theta_{m+1}}(m+1)
$$

is valid. We then get from (35) that

$$
\begin{aligned}
& W(m, x, i)=x^{\prime}\left(Q_{i}+G(m)^{\prime} R_{i} G(m)\right) x \\
& \quad+\mathrm{E}\left[x_{m+1}^{\prime} L_{\theta_{m+1}}(m+1) x_{m+1}+\omega_{\theta_{m+1}}(m+1) \mid \theta_{m}=i, x_{m}=x\right] .
\end{aligned}
$$

The right-hand side of the above identity is equal to

$$
\begin{aligned}
x^{\prime}\left(Q_{i}+G(m)^{\prime} R_{i} G(m)+A_{i}(m)^{\prime} \mathscr{E}_{i}(L(m+1)) A_{i}(m)\right) x+\mathscr{E}_{i}(\omega(m+1)) \\
+\mathrm{E}\left[\operatorname{tr}\left\{L_{\theta_{m+1}}(m+1) H_{i} w(m) w(m)^{\prime} H_{i}^{\prime}\right\} \mid \theta_{m}=i, x_{m}=x\right] .
\end{aligned}
$$

Since the last term in this expression is equal to $\operatorname{tr}\left\{\mathscr{E}_{i}(L(m+1)) H_{i} H_{i}^{\prime}\right\}$, we can conclude that

$$
\begin{aligned}
W(m, x, i)= & x^{\prime}\left(Q_{i}+G(m)^{\prime} R_{i} G(m)+A_{i}(m)^{\prime} \mathscr{E}_{i}(L(m+1)) A_{i}(m)\right) x \\
& +\mathscr{E}_{i}(\omega(m+1))+\operatorname{tr}\left\{\mathscr{E}_{i}(L(m+1)) H_{i} H_{i}^{\prime}\right\} \\
= & x^{\prime} L_{i}(m) x+\omega_{i}(m),
\end{aligned}
$$

which shows the result in (36) for $t=m$. This induction argument completes the proof of (36). The result of Lemma 2.1 then follows from the conditional expectation property together with (34) and (36).

Proof

(Proof that (18) and (19) hold). Recall from (8) that

$$
L_{i, \mathbf{G}}(k)=Q_{i}+G(k)^{\prime} R_{i} G(k)+\mathscr{L}_{i, \mathbf{G}}^{k}\left(L_{\mathbf{G}}(k+1)\right), \quad k=0, \ldots, N-1, \forall i \in \mathscr{S},
$$

with $L_{\mathbf{G}}(N)=F$, where the operator $\mathscr{L}_{\mathbf{G}}^{k}: \mathbb{S}^{r} \mapsto \mathbb{S}^{r}$ is defined as

$$
\mathscr{L}_{i, \mathbf{G}}^{k}(U):=\left(A_{i}+B_{i} G(k)\right)^{\prime} \mathscr{E}_{i}(U)\left(A_{i}+B_{i} G(k)\right), \forall i \in \mathscr{S}, U \in \mathbb{S}^{r} .
$$


We want to show that

$$
L_{\mathbf{G}}(k)-L_{\mathbf{K}}(k)=\delta_{\mathbf{G}}^{k}+\mathscr{L}_{\mathbf{K}}^{k}\left(L_{\mathbf{G}}(k+1)-L_{\mathbf{K}}(k+1)\right), \quad k=0, \ldots, N-1,
$$

where

$$
\delta_{i, \mathbf{G}}^{k}:=\left(G(k)-Z_{i}^{k}\right)^{\prime} \Lambda_{i, \mathbf{G}}^{k+1}\left(G(k)-Z_{i}^{k}\right)-\left(K(k)-Z_{i}^{k}\right)^{\prime} \Lambda_{i, \mathbf{G}}^{k+1}\left(K(k)-Z_{i}^{k}\right), \forall i \in \mathscr{S},
$$

with $\Lambda_{i, \mathbf{G}}^{k}:=R_{i}+B_{i}^{\prime} \mathscr{E}_{i}\left(L_{\mathbf{G}}(k)\right) B_{i}$ and $Z_{i}^{k}:=-\left(\Lambda_{i, \mathbf{G}}^{k+1}\right)^{-1} B_{i}^{\prime} \mathscr{E}_{i}\left(L_{\mathbf{G}}(k+1)\right) A_{i}$.

By simplifying the notation, we can evaluate $L_{\mathbf{G}}(k)-L_{\mathbf{K}}(k)$ as follows.

$$
L_{\mathbf{G}}=Q+G^{\prime} R G+(A+B G) \mathscr{E}\left(L_{\mathbf{G}}^{+}\right)(A+B G) .
$$

Thus,

$$
\begin{aligned}
L_{\mathbf{G}}-L_{\mathbf{K}}= & G^{\prime} R G-K^{\prime} R K+(A+B G) \mathscr{E}\left(L_{\mathbf{G}}^{+}\right)(A+B G) \\
& -(A+B K) \mathscr{E}\left(L_{\mathbf{K}}^{+}\right)(A+B K) .
\end{aligned}
$$

By completing the expression for a quadratic term, we get that

$$
\begin{aligned}
L_{\mathbf{G}}-L_{\mathbf{K}}= & G^{\prime} R G-K^{\prime} R K+(A+B K)^{\prime} \mathscr{E}\left(L_{\mathbf{G}}^{+}-L_{\mathbf{K}}^{+}\right)(A+B K) \\
& -K^{\prime} B^{\prime} \mathscr{E}\left(L_{\mathbf{G}}^{+}\right) B K-K^{\prime} B^{\prime} \mathscr{E}\left(L_{\mathbf{G}}^{+}\right) A-A^{\prime} \mathscr{E}(L \mathbf{G}) B K \\
& +G^{\prime} B^{\prime} \mathscr{E}\left(L_{\mathbf{G}}^{+}\right) B G+G^{\prime} B^{\prime} \mathscr{E}\left(L_{\mathbf{G}}^{+}\right) A+A^{\prime} \mathscr{E}\left(L_{\mathbf{G}}^{+}\right) B G \\
= & G^{\prime}\left(R+B^{\prime} \mathscr{E}\left(L_{\mathbf{G}}^{+}\right) B\right) G-K^{\prime}\left(R+B^{\prime} \mathscr{E}\left(L_{\mathbf{G}}^{+}\right) B\right) K \\
& +G^{\prime} B^{\prime} \mathscr{E}\left(L_{\mathbf{G}}^{+}\right) A+A^{\prime} \mathscr{E}\left(L_{\mathbf{G}}^{+}\right) B G \\
& -K^{\prime} B^{\prime} \mathscr{E}\left(L_{\mathbf{G}}^{+}\right) A-A^{\prime} \mathscr{E}\left(L_{\mathbf{G}}^{+}\right) B K+\mathscr{L}_{\mathbf{K}}\left(L_{\mathbf{G}}^{+}-L_{\mathbf{K}}^{+}\right) \\
= & G^{\prime} \Lambda_{\mathbf{G}}^{+} G-K^{\prime} \Lambda_{\mathbf{G}}^{+} K-G^{\prime} \Lambda_{\mathbf{G}}^{+} Z^{+}-\left(Z^{+}\right)^{\prime} \Lambda_{\mathbf{G}}^{+} G \\
& +K^{\prime} \Lambda_{\mathbf{G}}^{+} Z^{+}+\left(Z^{+}\right)^{\prime} \Lambda_{\mathbf{G}}^{+} K+\mathscr{L}_{\mathbf{K}}\left(L_{\mathbf{G}}^{+}-L_{\mathbf{K}}^{+}\right) \\
= & \left(G-Z^{+}\right)^{\prime} \Lambda_{\mathbf{G}}^{+}\left(G-Z^{+}\right)-\left(K-Z^{+}\right)^{\prime} \Lambda_{\mathbf{G}}^{+}\left(K-Z^{+}\right) \\
& +\mathscr{L}_{\mathbf{K}}\left(L_{\mathbf{G}}^{+}-L_{\mathbf{K}}^{+}\right) \\
= & \delta_{\mathbf{G}}+\mathscr{L}_{\mathbf{K}}\left(L_{\mathbf{G}}^{+}-L_{\mathbf{K}}^{+}\right) .
\end{aligned}
$$

The last equality in the above expression shows that (18) and (19) hold.

\section{REFERENCES}

1. E. Assunção, C. Q. Andrea, and M. C. M. Teixeira. $H_{2}$ and $H_{\infty}$-optimal control for the tracking problem with zero variation. IET Control Theory Appl., 1(3):682-688, 2007.

2. D. P. Bertsekas. Dynamic Programming and Optimal Control. Athena Scientific, 2007.

3. E. F. Costa and J. B. R. do Val. On the detectability and observability of continuous-time Markov jump linear systems. SIAM J. Control Optim., 41(4):1295-1314, 2002.

4. E. F. Costa, J. B. R. do Val, and M. D. Fragoso. A new approach to detectability of discrete-time Markov jump linear systems. SIAM J. Control Optim., 43(6):2132-2156, 2005.

5. E. F. Costa, A. N. Vargas, and J. B. R. do Val. Quadratic costs and second moments of jump linear systems with general Markov chain. Math. Control Signals Systems, 23(1):141-157, 2011.

6. O. L. V. Costa and W. L. de Paulo. Indefinite quadratic with linear costs optimal control of Markov jump with multiplicative noise systems. Automatica, 43:587-597, 2007.

7. O. L. V. Costa, M. D. Fragoso, and R. P. Marques. Discrete-Time Markovian Jump Linear Systems. SpringerVerlag, New York, 2005.

8. O. L. V. Costa and E. F. Tuesta. Finite horizon quadratic optimal control and a separation principle for Markovian jump linear systems. IEEE Trans. Automat. Control, 48, 2003.

9. J. B. R. do Val and T. Başar. Receding horizon control of jump linear systems and a macroeconomic policy problem. J. Econom. Dynam. Control, 23:1099-1131, 1999.

10. J. B. R. do Val, J. C. Geromel, and A. P. Gonçalves. The $H_{2}$ control for jump linear systems: Cluster observations of the Markov state. Automatica, 38:343-349, 2002. 
11. V. Dragan and T. Morozan. Exponential stability in mean square for a general class of discrete-time linear stochastic systems. Stoch. Anal. Appl., 26(3):495-525, 2008.

12. M. D. Fragoso and O. L. V. Costa. A separation principle for the continuous-time LQ-problem with Markovian jump parameters. IEEE Trans. Automat. Control, 55(12):2692-2707, 2010.

13. J. C. Geromel, A. P. C. Gonçalves, and A. R. Fioravanti. Dynamic output feedback control of discrete-time Markov jump linear systems through linear matrix inequalities. SIAM J. Control Optim., 48(2):573-593, 2009.

14. João P. Hespanha, Payam Naghshtabrizi, and Yonggang Xu. A survey of recent results in networked control systems. Proc. of IEEE Special Issue on Technology of Networked Control Systems, 95(1):138-162, Jan. 2007.

15. M. Khanbaghi, R. P. Malhame, and M. Perrier. Optimal white water and broke recirculation policies in paper mills via jump linear quadratic control. IEEE Trans. Automat. Control, 10(4):578-588, 2002.

16. A. Kojima and S. Ishijima. LQ preview synthesis: Optimal control and worst case analysis. IEEE Trans. Automat. Control, 44(2):352-357, 1999.

17. P. R. Kumar and P. Varaiya. Stochastic Systems: Estimation, identification and adaptive control. Prentice-Hall, Inc., New Jersey, 1986.

18. W. Leonhard. Control of Electrical Drives. Springer, 3rd edition, 2001.

19. X. Luan, F. Liu, and P. Shi. Finite-time stabilization of stochastic systems with partially known transition probabilities. J. Dyn. Syst. Meas. Control Trans. ASME., 133(1):14504-14510, 2011.

20. R. C. L. F. Oliveira, A. N. Vargas, J. B. R. do Val, and P. L. D. Peres. Robust stability, H2 analysis and stabilisation of discrete-time Markov jump linear systems with uncertain probability matrix. Internat. J. Control, 82(3):470 481, 2009.

21. C. L. Phillips and R. D. Harbor. Feedback Control Systems. Prentice Hall, 3rd edition, 1996.

22. A. Rubaai and R. Kotaru. Online identification and control of a DC motor using learning adaptation of neural networks. IEEE Trans. Industry Appl., 36(3):935-942, 2000.

23. M. Ruderman, J. Krettek, F. Hoffmann, and T. Bertram. Optimal state space control of DC motor. In Proc. 17th IFAC World Congress, pages 5796-5801, Seoul, Korea, 2008.

24. A. A. G. Siqueira and M. H. Terra. A fault tolerant manipulator robot based on $\mathrm{H} 2, \mathrm{H}$-infinity, and mixed $\mathrm{H} 2 / \mathrm{H}-$ infinity Markovian controls. IEEE/ASME Trans. Mechatronics, 14(2):257-263, 2009.

25. M. G. Todorov and M. D. Fragoso. On the robust stability, stabilization, and stability radii of continuous-time infinite Markov jump linear systems. SIAM J. Control Optim., 49:1171-1196, 2011.

26. A. N. Vargas and J. B. R. do Val. Average cost and stability of time-varying linear systems. IEEE Trans. Automat. Control, 55:714-720, 2010.

27. A. N. Vargas, W. Furloni, and J. B. R. do Val. Second moment constraints and the control problem of Markov jump linear systems. Numer. Linear Algebra Appl., 2012. doi:10.1002/nla.1849.

28. Y. Yin, P. Shi, and F. Liu. Gain scheduled PI tracking control on stochastic nonlinear systems with partially known transition probabilities. J. Franklin Inst., 348:685-702, 2011.

29. Y. Yin, P. Shi, F. Liu, and J. S. Pan. Gain-scheduled fault detection on stochastic nonlinear systems with partially known transition jump rates. Nonlinear Anal. Real World Appl., 13:359-369, 2012.

30. L. Zhang, E. Boukas, L. Baron, and H. R. Karimi. Fault detection for discrete-time Markov jump linear systems with partially known transition probabilities. Internat. J. Control, 83(8):1564-1572, 2010. 